\title{
Truncal impairment after stroke: clinical correlates, outcome and impact on ambulatory and functional outcomes after rehabilitation
}

Keng He Kong $^{1}$, MBBS, MRCP, Rathi d/o Ratha Krishnan ${ }^{1}$, MBBS, MPH

INTRODUCTION Good trunk performance is important for activities such as sitting and standing. In a cohort of patients with stroke, we sought to evaluate changes in trunk performance after stroke, establish factors correlated to trunk performance and assess the impact of trunk performance on discharge ambulatory and functional status.

METHODS This was a retrospective review of the data of patients with stroke admitted to Tan Tock Seng Hospital rehabilitation centre, Singapore, over a two-year period. Data analysed included the National Institutes of Health Stroke Scale (NIHSS), Montreal Cognitive Assessment (MOCA), Fugl-Meyer Assessment (FMA) of limb motor impairment and Functional Independence Measure-motor (FIM-motor) scores, which measures self-care ability. Trunk performance was assessed on the Trunk Impairment Scale (TIS).

RESULTS 577 patients with stroke (mean age $63.2 \pm 11.8$ years) were analysed. Truncal impairment was present in $96.4 \%$ of patients. Mean admission TIS score was $14.3 \pm 6.1$ and this improved to $17.2 \pm 5.2$ on discharge $(p<0.001)$. Admission TIS score was positively correlated with admission MOCA, FMA-upper limb and FMA-lower limb scores, and negatively correlated to NIHSS score and neglect. Admission TIS scores significantly predicted discharge FIM-motor scores $(p<0.001)$ and ambulatory status $(p<0.001)$.

CONCLUSION Truncal impairment was common and improvements in trunk performance were seen after rehabilitation. Trunk performance was significantly correlated to stroke severity, upper and lower limb motor power, cognition and neglect. As admission trunk performance predicted discharge functional and ambulatory status, it is recommended that trunk performance be evaluated for all patients with stroke.

Keywords: outcome, rehabilitation, stroke, trunk

\section{INTRODUCTION}

Good trunk performance is important for adequate performance of key activities such as sitting, standing and walking, as trunk muscles help to stabilise proximal body segments during voluntary movements of the extremities. ${ }^{(1)}$ Selective movements of the trunk are also required to maintain the centre of mass within the base of support and to maintain an upright posture during shifting of weight. Clinically, trunk performance can be assessed by tests ranging from a simple assessment of the patient's sitting balance to tests of balance (e.g. Berg's Balance Scale ${ }^{(2)}$ ), and more structured and detailed truncal activities on a standardised scale, such as the Trunk Impairment Scale (TIS). ${ }^{(3)}$ Previous studies have shown that impairments of trunk performance are common after stroke and they include impaired trunk muscle strength, decreased sitting balance and reduced truncal coordination..$^{(1,4,5)}$

The importance of understanding trunk performance after stroke is underlined by the finding that it has been shown to predict the ability to perform activities of daily living. ${ }^{(6,7)}$ In one study involving 102 patients with stroke, trunk performance on admission was even shown to be a stronger predictor of Barthel Index score at six months after stroke than that on admission. ${ }^{(8)}$ These findings suggest that if trunk performance could be improved early in the rehabilitation process, better functional improvement after stroke might be possible. In a randomised controlled study of 33 patients with stroke, patients who received six hours of additional truncal exercises had significantly better truncal function, standing balance and mobility when compared to control patients. ${ }^{(9)}$

Less well studied is the recovery of trunk performance after stroke and the neurological impairments associated with trunk performance. The above has relevant implications for the timing of rehabilitation intervention and prognostication of patient outcome. In a small study involving 32 patients with stroke, the time course of recovery of trunk performance, as measured on the TIS, was similar to that of the arm and leg, with most recovery occurring within the first three months after stroke. ${ }^{(10)}$

At the Tan Tock Seng Hospital (TTSH) rehabilitation centre, Singapore, as part of our centre's stroke database, we have been measuring truncal performance using the TIS for all patients with stroke admitted to the centre since 2014. This study on a cohort of patients with stroke admitted to our rehabilitation centre aimed to: (a) evaluate trunk performance before and after rehabilitation; (b) establish clinical factors associated with trunk performance; and (c) evaluate the impact of trunk performance on discharge ambulatory and functional status.

\section{METHODS}

All patients with stroke admitted to the rehabilitation centre at TTSH were prospectively included in the centre's database. Admission to the centre's stroke rehabilitation programme was

${ }_{1}^{1}$ Department of Rehabilitation Medicine, Tan Tock Seng Hospital, Singapore

Correspondence: Dr Kong Keng He, Senior Consultant, Department of Rehabilitation Medicine, Tan Tock Seng Hospital, 11 Jalan Tan Tock Seng, Singapore 308433. keng_he_kong@ttsh.com.sg 
evaluated by a rehabilitation physician and included the criteria: (a) significant impairment and disability that may improve with rehabilitation; and (b) ability to participate in at least two hours of therapy daily.

Data routinely captured in the centre's database included: (a) measures of stroke-related severity and impairments, which included stroke severity as measured on the National Institutes of Health Stroke Scale (NIHSS), ${ }^{(11)}$ cognition as assessed on the Montreal Cognitive Assessment (MOCA), ${ }^{(12)}$ motor impairment as measured on the Fugl-Meyer Assessment (FMA) ${ }^{(13)}$ and trunk performance as measured on TIS; ${ }^{(3)}$ (b) functional status as measured on the Functional Independence Measure (FIM); ${ }^{(14)}$ and (c) patient demographics, length of stay in rehabilitation and discharge disposition.

The TIS measured static sitting balance, dynamic sitting balance and coordination. Static sitting balance evaluated if a patient could keep a seated position, with both feet on the floor and their legs crossed. Dynamic sitting balance assessed selective lateral flexion, initiated from the shoulder and pelvic girdle. Finally, coordination evaluated selective rotation of the upper and lower part of the trunk against time. Scores of static sitting balance (range 0-7), dynamic sitting balance (range 0-10) and coordination (range $0-6$ ) were summated to give total scores with a range of $0-23$; the higher the score, the better the trunk performance. A score less than 23 suggested presence of truncal impairment. The TIS has been previously validated in patients with stroke. ${ }^{(3)}$

The FMA consisted of the FMA-upper limb and FMA-lower limb domains, and scores were in the range of 0-66 for FMAupper limb and 0-34 for FMA-lower limb. Higher scores were associated with better motor power.

The FIM is a validated and reliable tool for assessing ability to perform self-care and mobility in various rehabilitation settings. It consists of 18 categories grouped into two subscales - motor and cognition. Each category scored in the range of 1-7; the higher the score, the more independent the patient was in performing the task associated with the item.

As the TIS was only routinely measured for all patients with stroke beginning in 2014, for the purposes of this study, only data of patients admitted in the years 2014 and 2015 was analysed. The inclusion criteria were: (a) patients with a first stroke (ischaemic and haemorrhagic) as diagnosed radiologically either on computed tomography and/or magnetic resonance imaging of the brain; and (b) admission to rehabilitation within four weeks of stroke onset. Exclusion criteria were: (a) patients with recurrent stroke; and (b) patients who did not complete their rehabilitation programmes.

FMA, FIM and TIS were assessed within 72 hours and one week of rehabilitation admission and discharge, respectively, while NIHSS and MOCA scores were only assessed on rehabilitation admission.

Statistical analysis was carried out using IBM SPSS Statistics version 22.0 (IBM Corp, Armonk, NY, USA). All statistical tests were carried out at a $5 \%$ level of significance, so that $p<0.05$ was considered to be statistically significant. The $t$-test was used for comparison of means of continuous variables, Pearson correlation coefficient was used for correlation of continuous variables, and chi-square test was used for comparison of categorical variables.

The following analyses were performed: (a) comparison of TIS scores on admission to and discharge from rehabilitation; (b) correlation of measures of stroke severity and impairments with admission TIS scores; and (c) evaluation of the potential of admission TIS scores for predicting discharge ambulatory status and FIM-motor score.

The variables studied for correlation of measures of stroke severity to admission TIS scores included admission NIHSS, MOCA and FMA scores, neglect and age. Neglect was assessed based on relevant subscores of NIHSS and was defined as an NIHSS sensory extinction score $\geq 1$.

Ambulatory status was assessed on the FIM-walk score. Poor ambulators were defined as patients with FIM-walk score $\leq 4$ and good ambulators were patients with FIM-walk score $\geq 5$. An FIMwalk score of 5 implied either that the patient required standby supervision, cueing or coaxing to go a minimum of $150 \mathrm{ft}$ (50 m), or was able to walk only short distances (a minimum of $50 \mathrm{ft}$ or $15 \mathrm{~m}$ ) independently with or without a device.

For the prediction of discharge FIM-motor score, apart from admission TIS score, other variables analysed included age, neglect and admission FMA-total, NIHSS and MOCA scores. For (b) and (c), multivariate logistic regression analyses were performed if there were more than one significant independent variable on univariate logistic regression analysis.

The study was approved by the hospital's ethics committee.

\section{RESULTS}

Data of 577 patients with stroke who met the inclusion and exclusion criteria was analysed. Clinical characteristics and rehabilitation outcomes are shown in Table I. Mean age was 63.2 \pm 11.8 years, with a predominance of men $(65.0 \%)$. Ethnically, a majority of patients were Chinese $(78.0 \%)$ followed by Malay (12.5\%). Over two-thirds of patients had ischaemic stroke (69.0\%) and a majority of patients had unilateral weakness (96.5\%). Dysphasia and neglect were present in $121(21.0 \%)$ and 54 $(9.4 \%)$ patients, respectively. Mean lengths of stay in the acute facility and rehabilitation were $12.9 \pm 11.1$ days and $26.9 \pm 17.3$ days, respectively.

Only 21 (3.6\%) patients had full TIS scores on admission, indicating that a majority of patients had impaired trunk performance. Mean change in TIS scores from admission to discharge was $2.8 \pm 3.3$ and this was statistically significant $(p<0.001)$. Significant improvements were also noted in all subscales of the TIS $(p<0.001)$. Patients with longer lengths of rehabilitation stay had significantly lower admission TIS scores $(r=-0.45 ; p<0.001)$.

The results of analysis of variables associated with admission TIS score are given in Table II. Admission TIS scores were positively correlated to admission MOCA and FMA-upper limb and FMA-lower limb scores, but negatively correlated to admission NIHSS score and presence of neglect. Based on the 
Table I. Clinical characteristics and rehabilitation outcomes $(n=577)$.

\begin{tabular}{|c|c|c|c|}
\hline \multirow[t]{2}{*}{ Variable } & \multicolumn{2}{|c|}{ No. $(\%) / m e a n \pm$ standard deviation } & \multirow[t]{2}{*}{ p-value } \\
\hline & Admission & Discharge & \\
\hline Age (yr) & $63.2 \pm 11.8$ & - & - \\
\hline \multicolumn{4}{|l|}{ Gender } \\
\hline Male & $375(65.0)$ & - & - \\
\hline Female & $202(35.0)$ & - & - \\
\hline \multicolumn{4}{|l|}{ Ethnicity } \\
\hline Chinese & $450(78.0)$ & - & - \\
\hline Malay & $72(12.5)$ & - & - \\
\hline Indian & $36(6.3)$ & - & - \\
\hline Other & $19(3.3)$ & - & - \\
\hline \multicolumn{4}{|l|}{ Nature of stroke } \\
\hline Infarct & $398(69.0)$ & - & - \\
\hline Haemorrhage & $179(31.0)$ & - & - \\
\hline \multicolumn{4}{|l|}{ Side of stroke } \\
\hline Left & $274(47.5)$ & - & - \\
\hline Right & $283(49.0)$ & - & - \\
\hline Bilateral & $20(3.5)$ & - & - \\
\hline Length of stay in acute facility (day) & $12.9 \pm 11.1$ & - & - \\
\hline Length of stay in rehabilitation (day) & $26.9 \pm 17.3$ & - & - \\
\hline NIHSS score & $4.6 \pm 4.1$ & & - \\
\hline Neglect & $54(9.4)$ & - & - \\
\hline Dysphasia & $121(21.0 \%)$ & - & - \\
\hline MOCA score & $16.7 \pm 9.4$ & - & - \\
\hline TIS score & $14.3 \pm 6.1$ & $17.2 \pm 5.2$ & $<0.001$ \\
\hline Static balance & $5.9 \pm 2.1$ & $6.6 \pm 1.4$ & $<0.001$ \\
\hline Dynamic balance & $5.7 \pm 3.1$ & $7.0 \pm 2.8$ & $<0.001$ \\
\hline Coordination & $2.6 \pm 1.8$ & $3.4 \pm 1.9$ & $<0.001$ \\
\hline FMA-total score & $63.8 \pm 32.3$ & $74.2 \pm 29.2$ & $<0.001$ \\
\hline Upper limb & $42.0 \pm 23.9$ & $48.3 \pm 22.0$ & $<0.001$ \\
\hline Lower limb & $21.8 \pm 10.2$ & $25.9 \pm 9.3$ & $<0.001$ \\
\hline FIM-motor score & $45.4 \pm 15.6$ & $58.4 \pm 20.2$ & $<0.001$ \\
\hline
\end{tabular}

FIM: Functional Independence Measure; FMA: Fugl-Meyer Assessment; MOCA: Montreal Cognitive Assessment; NIHSS: National Institutes of Health Stroke Scale; TIS: Trunk Impairment Scale

value of the correlation coefficient, admission NIHSS and FMA scores were highly correlated, while admission MOCA score was moderately correlated to admission TIS scores. On multivariate logistic regression analysis, all variables remained significantly correlated to admission TIS scores and, together, they accounted for $52 \%$ of variance of the admission TIS scores.

Mean admission TIS score was significantly higher in patients with good discharge ambulatory outcome than in patients with poor discharge ambulatory outcome (16.5 \pm 5.2 vs. $11.2 \pm 7.3$; $p<$ 0.001). Median admission TIS score was 14 , and the 25 th and 75 th percentile scores were 11 and 19, respectively. We compared the predictive potential of these different admission TIS scores and found the percentage of patients achieving good discharge ambulatory status with admission TIS scores $\geq 11, \geq 14$ and $\geq 19$ were $63.9 \%$, $68.8 \%$ and $72.3 \%$, respectively. In the 21 patients with full admission TIS scores, 19 (90.5\%) achieved good discharge ambulatory status.

Results of univariate logistic regression analysis of variables predicting discharge FIM-motor scores are shown in Table III.
Age, neglect, and admission scores of NIHSS, MOCA, TIS and FMA-total were all significant predictors of discharge FIM-motor scores. However, only age and admission MOCA, TIS and FMA-total scores remained significant predictors on multiple logistic regression analysis, with admission TIS score being the most important, as it showed the highest beta value of 0.23 (Table IV).

\section{DISCUSSION}

In this retrospective study of 577 patients with stroke, impaired trunk performance was common, occurring in 556 (96.4\%) patients. The relatively low mean admission TIS score of 14.3 suggested that most patients had significant truncal impairment. Breakdown of the admission TIS scores revealed almost full static sitting balance scores (mean score 5.9 out of a maximum score of 7) but low dynamic sitting balance (mean score 5.7 out of a maximum score of 10) and coordination (mean score 2.6 out of a maximum score of 6 ) scores. This was not surprising, as tasks 
Table II. Univariate logistic regression analysis of variables associated with admission TIS scores.

\begin{tabular}{|lll|}
\hline Variable & Admission TIS score & p-value \\
\hline Age & -0.03 & 0.45 \\
\hline Admission NIHSS score & -0.57 & $<0.001$ \\
\hline Admission MOCA score & 0.32 & $<0.001$ \\
\hline Admission FMA score & 0.68 & $<0.001$ \\
\hline Upper limb & 0.62 & $<0.001$ \\
\hline Lower limb & 0.68 & $<0.001$ \\
\hline Neglect* & & $<0.001$ \\
\hline Yes & $8.6 \pm 7.9$ & \\
\hline No & $4.8 \pm 5.5$ & \\
\hline
\end{tabular}

*Data presented as mean \pm standard deviation. FMA: Fugl-Meyer Assessment; MOCA: Montreal Cognitive Assessment; NIHSS: National Institutes of Health Stroke Scale; SD: standard deviation; TIS: Trunk Impairment Scale

on the last two subscales challenge the trunk more than tasks on static sitting balance.

Significant improvements in truncal performance were noted after rehabilitation, as evidenced by a mean gain of $2.8 \pm$ 3.3 points on the TIS. This gain was very close to the minimal clinically important difference for TIS, which has been reported to be 3.5 in subacute stroke. ${ }^{(15)}$ The number of patients, in our study, with a mean TIS gain of over 3.5 points was 160 (27.7\%).

Admission TIS scores were significantly correlated to admission NIHSS, MOCA, FMA-upper and FMA-lower limb scores and neglect. Patients with lower admission TIS scores were more likely to have a severe stroke, cognitive impairment, greater upper and lower limb weakness, and neglect. Together, these factors accounted for $52 \%$ of variance of the admission TIS score. To the best of our knowledge, in the only previous study that looked at variables correlated to admission TIS score, van Nes et al reported that in a cohort of 78 patients assessed within two weeks of stroke onset, admission TIS score was independently correlated to age, limb motor power and neglect, and these three variables accounted for $65 \%$ of variance of the admission TIS score. ${ }^{(16)}$ Patients in this earlier study were significantly older, with a mean age of 71.2 years, compared to 63.2 years in our study. This age disparity may explain why age was not a significant correlate of admission TIS score in our study.

The association between truncal impairment and upper and lower limb weaknesses has been reported previously. In a small study involving 32 patients with stroke from one week to six months after stroke onset, Verheyden et al showed that TIS score was closely correlated to upper and lower limb FMA scores at one week, one month, three months and six months after stroke onset, suggesting that truncal and limb recovery have similar recovery trajectories. ${ }^{(10)}$

Patients with neglect have decreased awareness of the affected side and this is associated with deviation of the trunk toward the affected side and, in some patients, contraversive pushing, a condition in which patients seem to actively push away from the non-paretic side. ${ }^{(17,18)}$ Hence, the significant correlation between truncal impairment and neglect was not surprising.
Table III. Univariate logistic regression analysis of variables associated with discharge FIM-motor scores.

\begin{tabular}{|lll|}
\hline Variable & $\begin{array}{l}\text { Discharge } \\
\text { FIM-motor score }\end{array}$ & p-value \\
\hline Age & $r=-0.16$ & $<0.001$ \\
\hline Admission NIHSS score & $r=-0.31$ & $<0.001$ \\
\hline Admission MOCA score & $r=0.34$ & $<0.001$ \\
\hline Admission TIS score & $r=0.42$ & $<0.001$ \\
\hline Admission FMA-total score & $r=0.35$ & $<0.001$ \\
\hline Neglect* & & $<0.001$ \\
\hline Yes & $46.2 \pm 21.0$ & \\
\hline No & $59.6 \pm 19.5$ & \\
\hline
\end{tabular}

*Data presented as mean \pm standard deviation. FIM: Functional Independence Measure; FMA: Fugl-Meyer Assessment; MOCA: Montreal Cognitive Assessment; NIHSS: National Institutes of Health Stroke Scale; SD: standard deviation; TIS: Trunk Impairment Scale

Table IV. Multivariate logistic regression analysis of variables associated with discharge FIM-motor scores.

\begin{tabular}{|llll|}
\hline Variable & B & p-value & $\mathbf{9 5 \%} \mathbf{C l}$ \\
\hline Age & -0.19 & $<0.001$ & -0.46 to -0.20 \\
\hline Admission MOCA score & 0.19 & $<0.001$ & 0.27 to 0.65 \\
\hline Admission TIS score & 0.23 & $<0.001$ & 0.47 to 1.16 \\
\hline Admission FMA-total score & 0.17 & 0.002 & 0.04 to 0.17 \\
\hline
\end{tabular}

$\mathrm{Cl}$ : confidence interval; FIM: Functional Independence Measure; FMA: Fugl-Meyer Assessment; MOCA: Montreal Cognitive Assessment; TIS:Trunk Impairment Scale

To the best of our understanding, cognitive impairment, as an independent correlate of truncal performance, has not been reported previously. One possible reason is that in some studies, patients with significant cognitive impairments were intentionally excluded out of concern about the ability of these patients to follow test protocols, which included performance on the TIS. ${ }^{(1,10,19)}$ In our study, the TIS was performed on all patients, with or without cognitive impairment, and it was possible that those with significant cognitive impairments were more likely to be unable to follow instructions on the TIS, thus resulting in lower TIS scores. This could have possibly contributed to our finding of cognitive impairment as a significant correlate of trunk performance.

The finding that truncal impairment on admission was a significant predictor of discharge functional and ambulatory status was consistent with results of previous studies. ${ }^{(5-7,19,20)}$ Patients with good discharge ambulatory status, on average, scored 5 points more on the admission TIS score when compared with poor discharge ambulators. The strength of admission TIS score in predicting discharge ambulatory status was also reflected in the finding that patients with admission TIS scores of 14 or higher had almost a $70 \%$ probability of achieving good discharge ambulatory status, and those with full admission TIS scores had a 90.4\% probability of achieving good discharge ambulatory status. This information is particularly useful, as it helps guide patients and caregivers on the expected level of ambulatory status that could be achieved by the patient at the time of discharge and the need for continued ambulatory training. 
As a predictor of discharge functional status, the admission TIS score was more important than other clinically relevant predictors, including age and limb motor power. This finding is of particular relevance, as recent studies have shown the positive impact of specific truncal exercises for improving trunk function, balance and mobility in patients with stroke. ${ }^{(9,21,22)}$ Haruyama et al, in an assessor-blinded, randomised controlled trial of 32 participants in a stroke rehabilitation ward, showed that participants who received additional core stability training in lieu of conventional therapy had better scores on the TIS, Timed Up-and-Go test and Functional Ambulation Category. ${ }^{(22)}$

This study had several limitations. Firstly, patients in our cohort were selected for admission to rehabilitation based on their ability, and this selection bias limited the generalisability of our results. Secondly, the retrospective nature of the study meant that other clinical variables that may have potential impact on study outcomes (e.g. site of stroke and mood) could not be evaluated. Furthermore, as the NIHSS was measured on admission to rehabilitation rather than within 72 hours of stroke onset, it may not accurately reflect stroke severity, as NIHSS scores tend to improve over time. On the other hand, the study sample size of 577 patients is the largest of all existing studies investigating trunk performance in patients with stroke.

In conclusion, in this cohort of patients with stroke admitted to our rehabilitation centre, truncal impairment was common and improvements in trunk performance were seen after rehabilitation. Trunk performance was significantly correlated to stroke severity, upper and lower limb motor power, cognition, and neglect. As admission trunk performance predicts discharge functional and ambulatory status, it is recommended that trunk performance be evaluated for all patients with stroke and, in those with impaired trunk performance, truncal exercises be considered to optimise trunk performance.

\section{REFERENCES}

1. Verheyden G, Vereeck L, Truijen S, et al. Trunk performance after stroke and the relationship with balance, gait and functional ability. Clin Rehabil 2006; 20:451-8.

2. Berg K, Wood-Dauphinee S, Williams JI, et al. Measuring balance in the elderly: validation of an instrument. Can J Pub Health 1992; 83:S7-11.
3. Verheyden G, Nieuwboer A, Mertin J, et al. The Trunk Impairment Scale: a new tool to measure motor impairment of the trunk after stroke. Clin Rehabil 2004; 18:326-34.

4. Bohannon RW, Cassidy D, Walsh S. Trunk muscle strength is impaired multidirectionally after stroke. Clin Rehab 1995; 9:47-51.

5. Ryerson S, Byl NN, Brown DA, Wong RA, Hidler JM. Altered trunk position sense and its relation to balance functions in people post-stroke. J Neurol Phys Ther 2008; 32:14-20.

6. Franchignoni FP, Tesio L, Ricupero C, Martino MT. Trunk control test as an early predictor of stroke rehabilitation outcome. Stroke 1997; 28:1382-5.

7. Hsieh CL, Sheu CF, Hsueh IP, Wang CH. Trunk control as an early predictor of comprehensive activities of daily living function in stroke patients. Stroke 2002; 33:2626-30.

8. Verheyden G, Nieuwboer A, De Wit L, et al. Trunk performance after stroke: an eye catching predictor of functional outcome. J Neurol Neurosurg Psychiatry 2007; 78:694-8.

9. Saeys W, Vereeck L, Truijen S, et al. Randomized controlled trial of truncal exercises early after stroke to improve balance and mobility. Neurorehabil Neural Repair 2012; 26:231-8.

10. Verheyden G, Nieuwboer A, De Wit L. Time course of trunk, arm, leg, and functional recovery after ischemic stroke. Neurorehabil Neural Repair 2008; 22:173-9.

11. Brott $\mathrm{T}$, Adams HP Jr, Olinger $\mathrm{CP}$, et al. Measurements of acute cerebral infarction: a clinical examination scale. Stroke 1989; 20:864-70.

12. Nasreddine ZS, Phillips NA, Bédirian V, et al. The Montreal Cognitive Assessment, MoCA: a brief screening tool for mild cognitive impairment. J Am Geriatr Soc 2005; 53:695-9.

13. Fugl-Meyer AR, Jääskö L, Leyman I, Olsson S, Steglind S. The post-stroke hemiplegic patient. 1. a method for evaluation of physical performance. Scand J Rehabil Med 1975; 7:13-31.

14. Keith RA, Granger CV, Hamilton BB, Sherwin FS. The functional independence measure: a new tool for rehabilitation. Adv Clin Rehabil 1987; 1:6-18.

15. Monticone M, Ambrosini E, Verheyden G, et al. Development of the Italian version of the trunk impairment scale in subjects with acute and chronic stroke. Cross-cultural adaptation, reliability, validity and responsiveness. Disabil Rehabil 2017; 10:1-8.

16. van Nes IJ, van der Linden S, Hendricks HT, et al. Is visuospatial hemineglect really a determinant of postural control following stroke? An acute-phase study. Neurorehabil Neural Repair 2009; 23:609-14

17. Pérennou DA, Leblond C, Amblard B, et al. Transcutaneous electric nerve stimulation reduces neglect-related postural instability after stroke. Arch Phys Med Rehabil 2001; 82:440-8.

18. Taylor D, Ashburn A, Ward CD. Asymmetrical trunk posture, unilateral neglect and motor performance following stroke. Clin Rehabil 1994; 8:48-53.

19. Masiero S, Avesani R, Armani M, Verena P, Ermani M. Predictive factors for ambulation in stroke patients in the rehabilitation setting: a multivariate analysis. Clin Neurol Neurosurg 2007; 109:763-9.

20. Duarte E, Marco E, Muniesa JM, et al. Trunk control test as a functional predictor in stroke patients. J Rehabil Med 2002; 34:267-72.

21. Cabanas-Valdés R, Bagur-Calafat $C$, Girabent-Farrés $M$, et al. The effect of additional core stability exercises on improving dynamic sitting balance and trunk control for subacute stroke patients: a randomized controlled trial. Clin Rehabil 2016; 30:1024-33.

22. Haruyama K, Kawakami M, Otsuka T. Effect of core stability training on trunk function, standing balance, and mobility in stroke patients. Neurorehabil Neural Repair 2017; 31:240-9. 